\title{
Arrhythmogenic right ventricular dysplasia masquerading as right ventricular outflow tract tachycardia
}

\author{
Daniel Pop-Mandru, Gabriel Cismaru, Dana Pop, Dumitru Zdrenghea
}

\begin{abstract}
Introduction: Ventricular tachycardia is a frequent complication in patients affected by arrhythmogenic right ventricular dysplasia. The disease is characterized by malignant ventricular arrhythmias with poor outcome, right ventricular dilation and replacement of the myocardium with fatty and fibrous tissue. On the other hand, right ventricular outflow tract tachycardia does not appear to have any significant effect on cardiac mortality as it arises in the absence of any structural heart disease. Case Report: Herein, we discussed a 62-year-old female patient with ventricular tachycardia that suggested right ventricular outflow tract tachycardia. Based on echocardiography, electrophysiological study and magnetic resonance imaging the final diagnosis was arrhythmogenic right ventricular dysplasia. During electrophysiological study two ventricular
\end{abstract}

Daniel Pop-Mandruㅜㄹ, Gabriel Cismaru², Dana Pop ${ }^{3}$, Dumitru Zdrenghea ${ }^{4}$

Affiliations: ${ }^{1} \mathrm{MD}$, Department of Cardiology, Rehabilitation Hospital, Iuliu Hatieganu University of Medicine and Pharmacy, Cluj-Napoca, Romania; ${ }^{2} \mathrm{MD}$, Electrophysiological laboratory, Department of Cardiology, Rehabilitation Hospital, Iuliu Hatieganu University of Medicine and Pharmacy, Cluj-Napoca, Romania; ${ }^{3} \mathrm{MD}$, $\mathrm{PhD}$, Head of didactic activity, Department of Cardiology, Rehabilitation Hospital, Iuliu Hatieganu University of Medicine and Pharmacy, Cluj-Napoca, Romania; ${ }^{4} \mathrm{MD}$, $\mathrm{PhD}$, Head of department, Department of Cardiology, Rehabilitation Hospital, luliu Hatieganu University of Medicine and Pharmacy, Cluj-Napoca, Romania.

Corresponding Author: Gabriel Cismaru, Cardiology Department, Rehabilitation Hospital No 46-50 Viilor Street Cluj-Napoca Cluj, Romania 400347; Ph: (40)264 207021; Fax: (40)264453131; Email:gabi_cismaru@yahoo.com

Received: 12 September 2013

Accepted: 04 October 2013

Published: 01 April 2014 tachycardias were induced, one hemodynamically stable and one with hemodynamic deterioration. The patient received an implantable cardioverterdefibrillator for prevention of sudden cardiac death. Conclusion: Electrophysiological study in combination with other diagnostic tools such as magnetic resonance imaging, echocardiography and 12-lead electrocardiography is useful for the differential diagnosis between arrhythmogenic right ventricular dysplasia and right ventricular outflow tract tachycardia and contributes to decision of implantable cardioverter defibrillators implantation.

Keywords: Ventricular tachycardia, Electrophysiological study, Arrhythmogenic right ventricular dysplasia, Implantable cardiac defibrillator (ICD)

\section{How to cite this article}

Pop-Mandru D, Cismaru G, Pop D, Zdrenghea D. Arrhythmogenic right ventricular dysplasia masquerading as right ventricular outflow tract tachycardia. International Journal of Case Reports and Images 2014;5(4):314-319.

doi:10.5348/ijcri-201454-CI-10011

\section{INTRODUCTION}

When the ventricular tachycardia (VT) exhibits a left bundle branch block (LBBB) pattern with an inferior axis, differential diagnosis must be made between arrhythmogenic right ventricular dysplasia (ARVD) and right ventricular outflow tract tachycardia (RVOT).

An ARVD is a disease that affects predominantly the right ventricle and is characterized by life-threatening malignant ventricular arrhythmias. It involves replacement of myocardium with fatty and fibrous tissue. 
This myocardial damage is associated with ventricular tachycardia and increased risk of sudden cardiac death [1]. Recommendations concerning the diagnosis and management of patients with ARVD were developed. The diagnosis is difficult and is currently based on electrocardiography (ECG), echocardiography, magnetic resonance imaging (MRI) scan, and right ventricular biopsy [2].

Idiopathic VT from RVOT is monomorphic and generally not familial. It shows specific morphologic features: LBBB morphology and QRS axis directed inferiorly. Echocardiography and MRI scan show normal RV and RVOT although small anatomical changes have been demonstrated during MRI examination [3, 4].

The difference between ARVD and RVOT is important when discussing prognosis and management options. An RVOT is considered to be curative with radiofrequency catheter ablation. This is the first line treatment for symptomatic patients [3]. The role of ablation in ARVD is more limited with lower acute success rates and with high later recurrences. The progressive nature of the disease and the risk of sudden cardiac death by severe ventricular arrhythmias make the implantable cardioverter defibrillators (ICD) implantation the principal recommendation in this group of patients.

\section{CASE REPORT}

A 62-year-old female was presented with palpitations in a district hospital and 12-lead ECG revealed wide QRS complex tachycardia suggesting RVOT. She was referred to our department for electrophysiological study and ablation. During the last month, she presented similar episodes with palpitations and dizziness. An LBBB morphology with inferior axis suggested RVOT (Figure 1). On admission, the patient had no sign of cardiac failure. No previous similar medical history was found in her family. On physical examination, her blood pressure was $130 / 70 \mathrm{mmHg}$. Neither moist nor dry rale could be auscultated in her lungs. Heart rate was $70 \mathrm{bpm}$. The systolic cardiac murmurs auscultated at the tricuspid valve was $2 / 6 \sim 3 / 6$. She had no signs of pitting Oedema at lower extremities. The transaminase level was normal including the renal function and the ionogram. Her chest X-ray showed mild increase of transverse cardiac diameter with uplifted apex and rounded left heart border (Figure 2). Electrocardiography demonstrated normal sinus rhythm with a heart rate of $77 \mathrm{bpm}$, incomplete right bundle branch block (RBBB), right-axis deviation, low voltage in the leads of the extremities, ventricular extrasystoles (with RBBB morphology), and T-wave inversion in leads $\mathrm{V}_{1}$ through $\mathrm{V}_{3}$ (Figure 3). Epsilon wave was apparent in leads V1 and V2. (Figure 4). Twodimensional echocardiography showed enlargement of right ventricle ( $40 \mathrm{~mm}$ from parasternal great axis), with reduced right ventricular function: (low TAPSE value and $\mathrm{S}$-wave in TDI at the free lateral wall of the right ventricle), associated with right atrium enlargement and severe tricuspid valve insufficiency. Further echocardiographic findings were a grade II mitral valve insufficiency and left ventricular ejection fraction of 50\% (Figure 5).

After signing a consent form, the patient underwent an electrophysiological study under cover of antiarrhythmic drugs which revealed two types of VT induced by ventricular programmed stimulation. The first VT associated pre-syncope and was stopped by ventricular overdrive (Figures 6-8). The second VT was rapid with a cycle length of $300 \mathrm{~ms}$, evoluated with syncope and
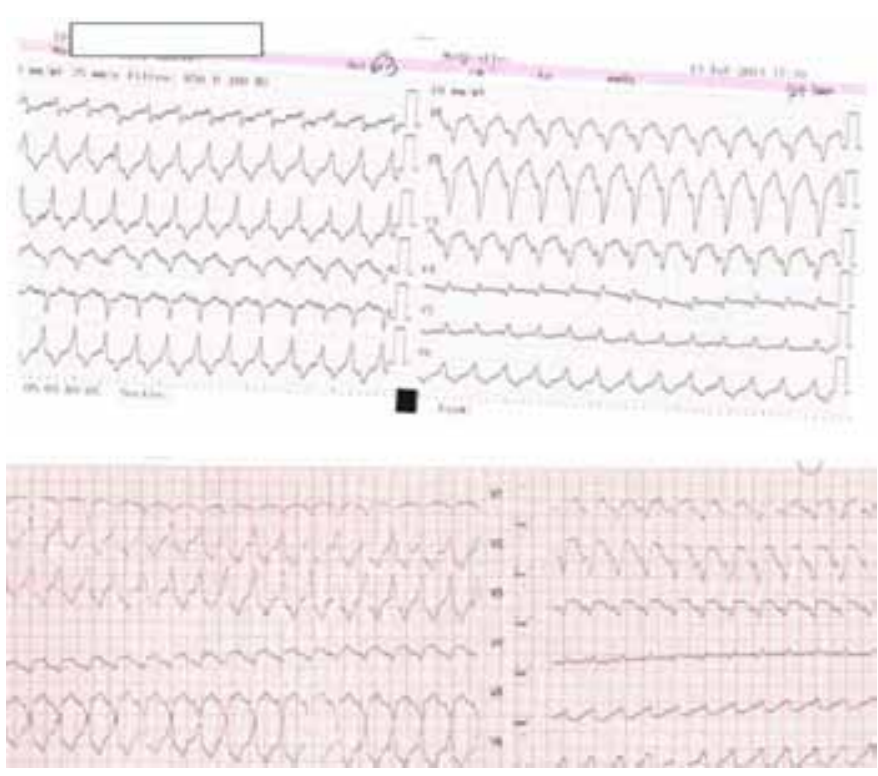

Figure 1: Recording of 12-lead electrocardiogram during four hours lasting tachycardia, showing: large QRS complex tachycardia with a left bundle branch block morphology and inferior axis; heart rate of $165 \mathrm{bpm}$. Positivity in leads II, II and avF suggests upper part of the right ventricle (right ventricular outflow tract) as origin of the ventricular tachycardia.

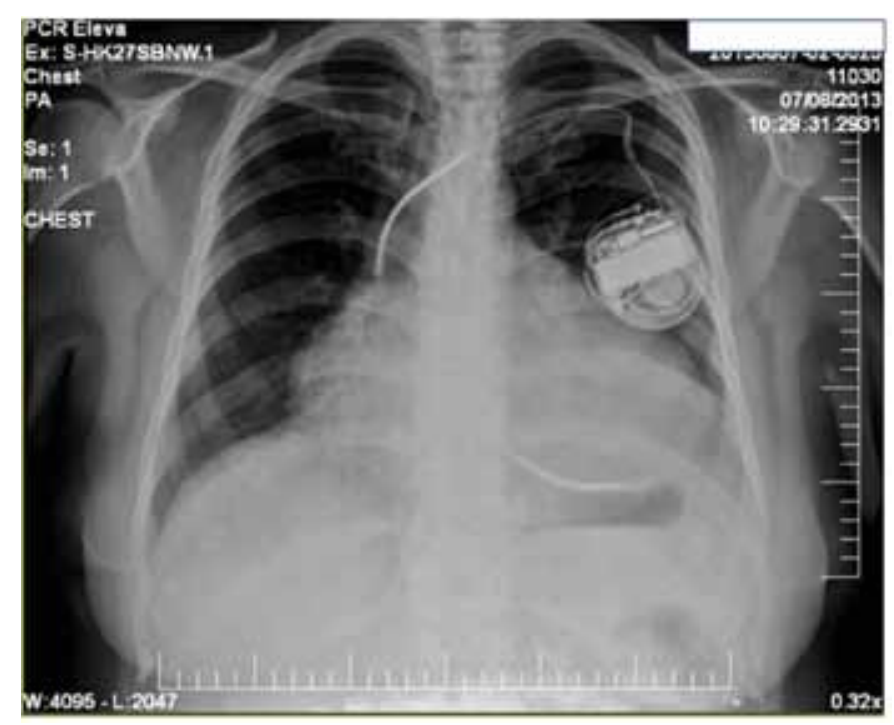

Figure 2: Chest X-ray in posterior-anterior view showing enlargement of the cardiac silhouette with a prominent right and left ventricular contour and with an automatic implantable cardioverter-defibrillator in the left pectoral area. 


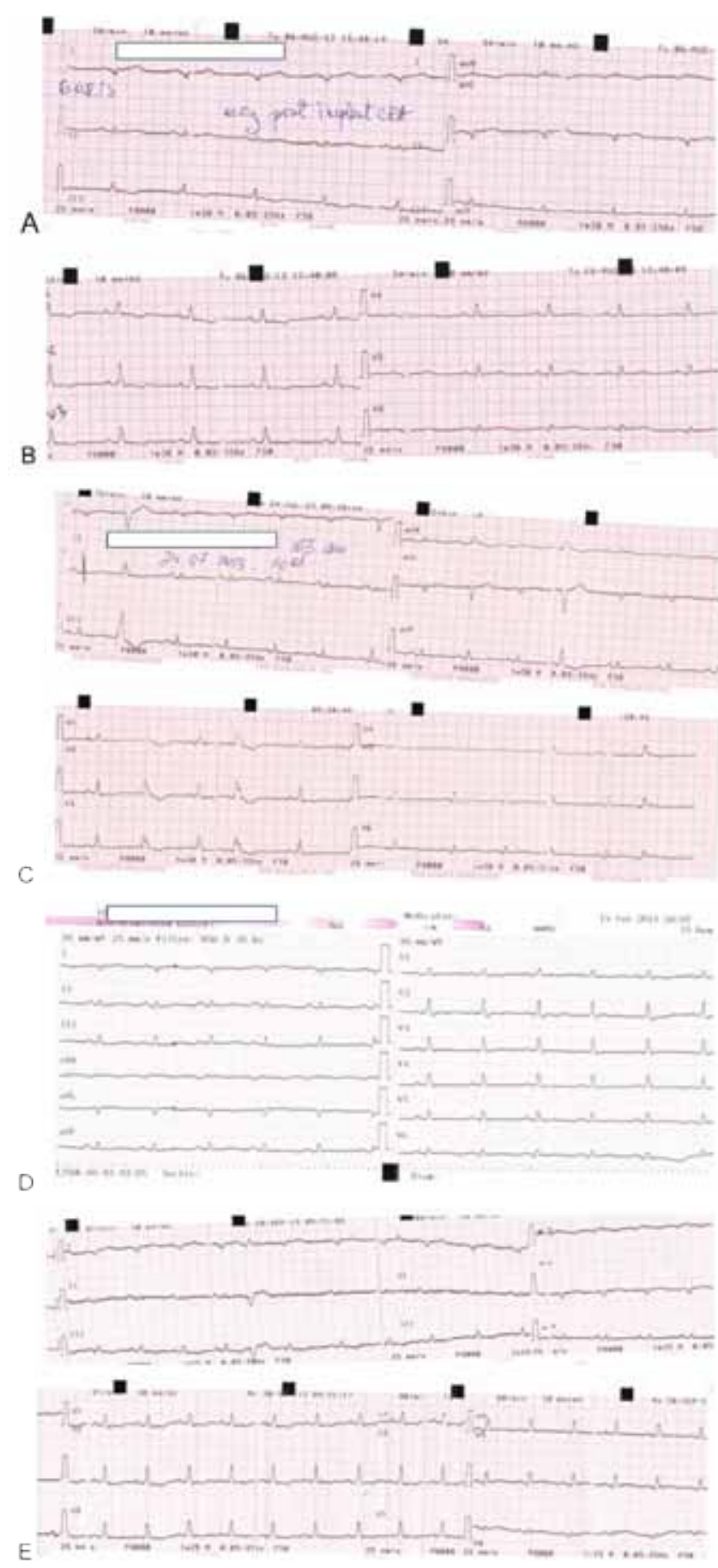

Figure 3: (A) Electrocardiography during admission to our hospital showing sinus rhythm, right-axis deviation, low voltage of the extremities, (B) incomplete right bundle branch block, $\mathrm{T}$-wave inversion in leads $\mathrm{V}_{1}$ to $\mathrm{V}_{3}$, (C) ventricular extrasystoles with a right bundle branch block appearance, (D) Limb lead misplacement can be suspected based on negative QRS complex on the Figure $3 \mathrm{~A}$ in leads D1 and avL. But actually the $\mathrm{P}$ wave is positive confirming good position of the leads. In fact extreme dilation of the right ventricle changed the QRS electrical axis to the right, and (E) One month after defibrillator implantation the outpatient control shows the same aspect in the limb leads and precordial leads. Concerning the positivity of the QRS complex from $\mathrm{V}_{1}$ to $\mathrm{V}_{5}$, this is also due to extreme dilation of the right ventricle and displacement of the interventricular septum to the left. This is why depolarization wavefront circulates towards V1 and positivates this lead. necessitated electrical shock for restoration of sinus rhythm (Figure 9 and Figure 10). No isoproterenol was administrated because VTs were induced during basal state.

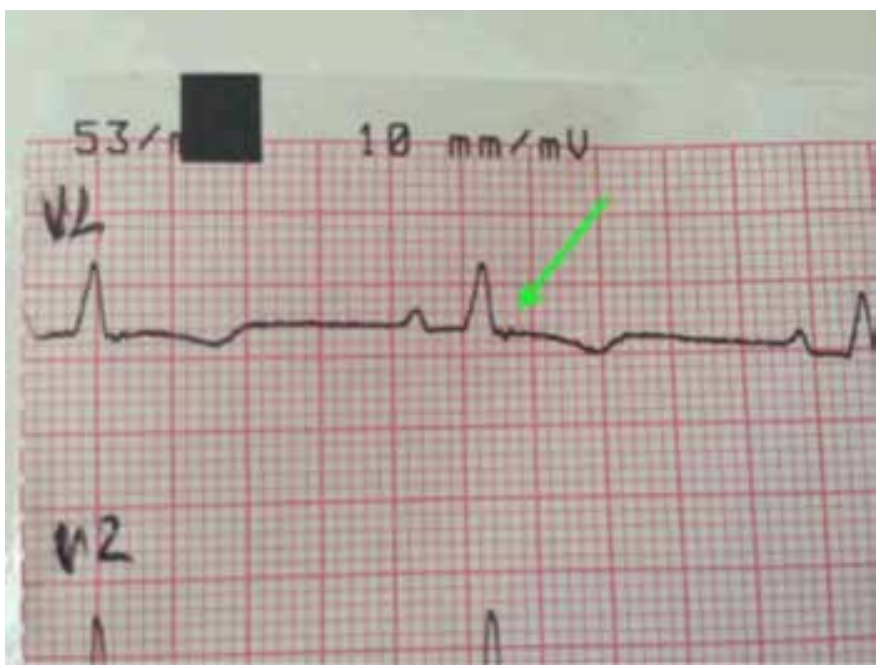

Figure 4: Presence of the epsilon wave at the end of the QRS complex in V1 lead.
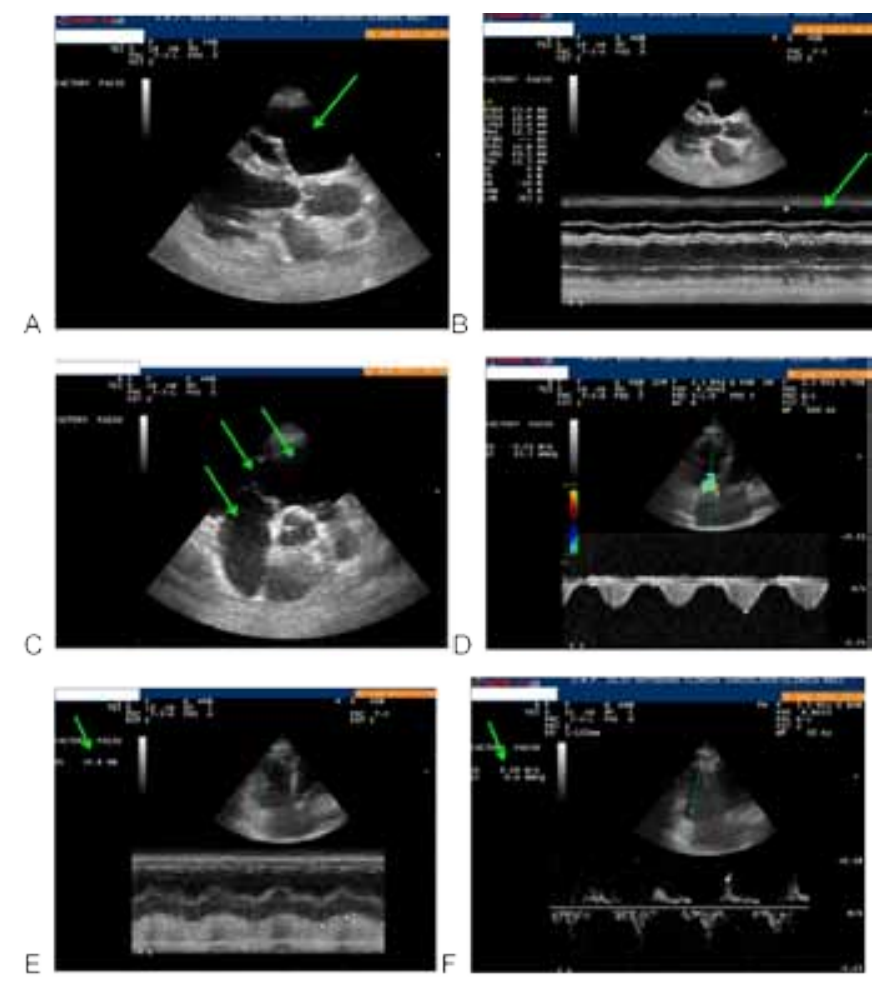

Figure 5: (A) Echocardiographic image in the parasternal long axis view showing dilation of the right ventricle in twodimensional mode, (B)in M-mode, (C) parasternal short axis at the level of great arteries showing, right atrial, right ventricular and right ventricular outflow tract dilation, (D) apical four chambers: tricuspid regurgitation in the doppler view, (E) apical 4 chambers showing reduced right ventricular function with low TAPSE at the lateral free wall tricuspid anulus, and (F) reduced right ventricular function demonstrated by reduced amplitude of the $\mathrm{S}$ wave in tissue Doppler imaging. 


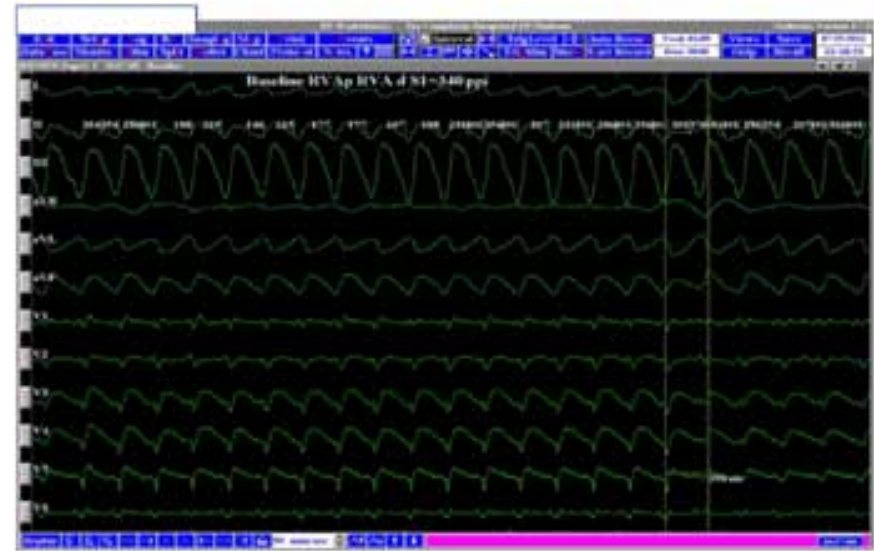

Figure 6: Ventricular tachycardia (VT1) induced during electrophysiological study. 12-lead electrocardiography shows a left bundle branch block QRS shape, negative in inferior leads (II, III and avF) and predominantly negative QRS complex in V2 to V6 suggesting origin of the tachycardia at the apex of the right ventricle.

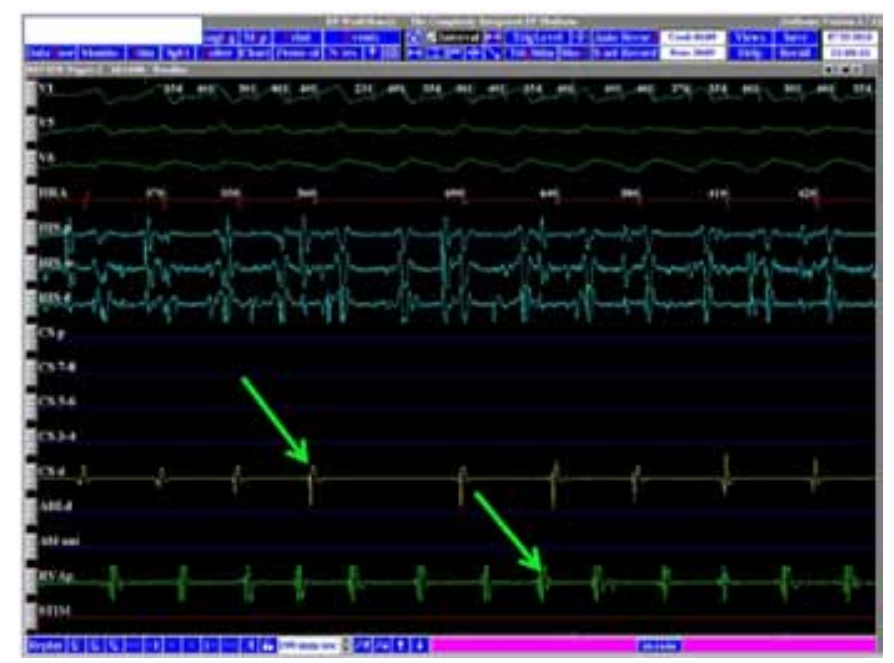

Figure 7: External and intracardiac electrograms during VT1 with: leads V1, V5 and V6 and electrograms from: high right atrium (HRA), His bundle proximal (His p), medium (His m) and distal (His d), coronary sinus - only the distal pole (CS d) being shown- and right ventricular apex (RVap). The ventricular electrogram (RVap) is concomitant with the QRS complex, but atrial electrograms (HRA) are dissociated from the ventricle confirming ventricular tachycardia.

Cardiac MRI scan presented the typical manifestation of ARVD which showed enlargement of right ventricle with aneurysmatic protrusion of the right ventricular free wall as well as dyskinetic areas of the outflow tract, without enlargement of left cavities. The wall of right ventricular outflow tract, the free wall of right ventricle and apex had fatty tissue infiltration.

Despite medical therapy with amiodarone and betablockers we could induce VTs during electrophysiological study, so finally she underwent ICD implantation. At one month outclinic control no spontaneous arrhythmia was detected by the ICD under $400 \mathrm{mg}$ amiodarone and 100 mg metoprolol.

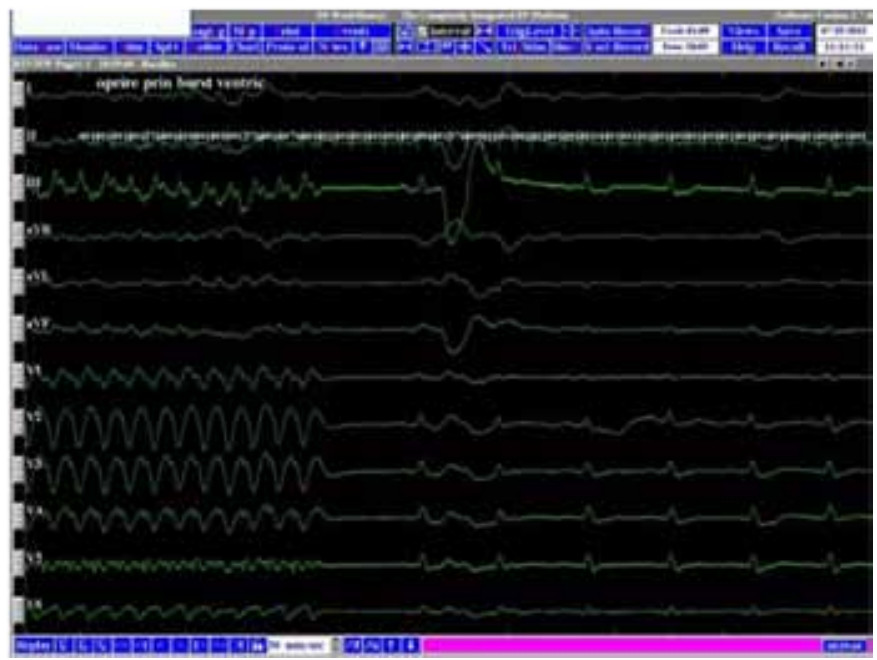

Figure 8: Ventricular tachycardia being stopped during electrophysiological study by ventricular overdrive.

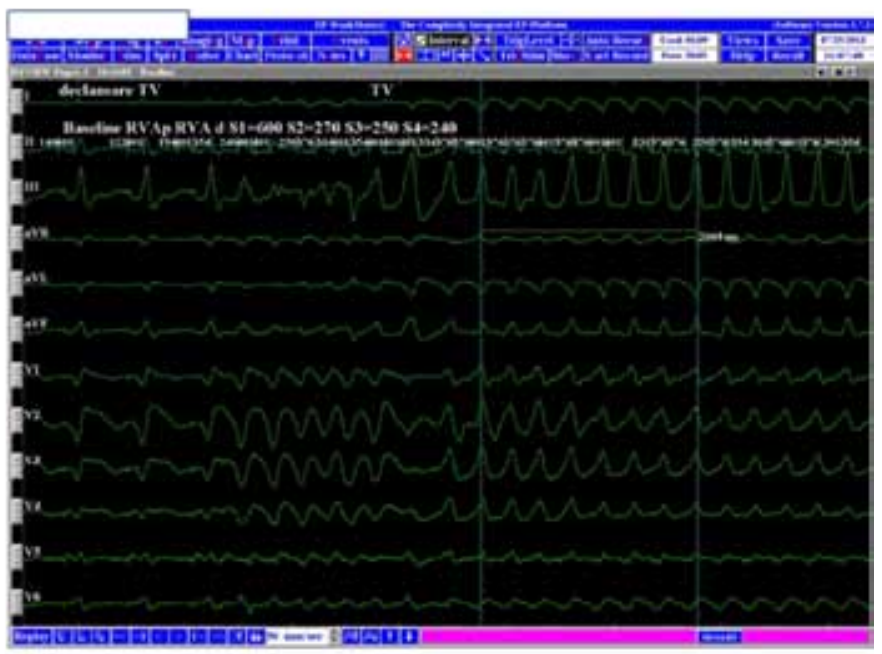

Figure 9: Induction of VT2 by ventricular extra stimuli. VT2 has a right bundle branch block appearance, with positive QRS complex in inferior leads: II, II avF, negative in lateral leads: D1 and avL; and positive from V1 to V4 in precordial leadssuggesting origin in the upper part of the right ventricle (e.g., peri-tricuspid area).

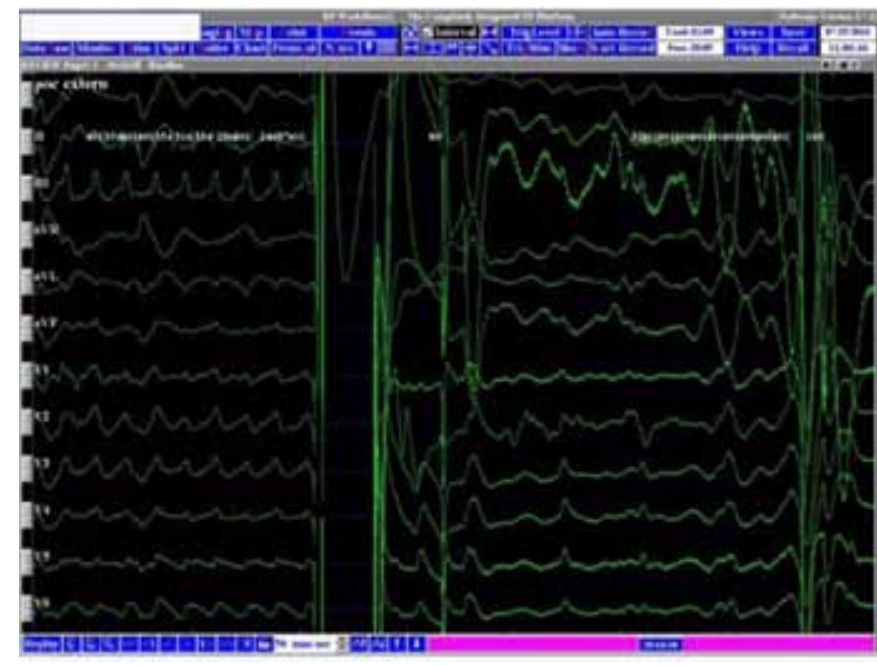

Figure 10: VT2 being stopped by an electrical shock. 


\section{DISCUSSION}

Most of the right ventricular tachycardias show a LBBB morphology with an inferior axis. The differential diagnosis is either idiopathic RVOT or ARVD. The diagnosis of ARVD is based on task force criteria, regarding: 12-lead ECG signs, combined with structural abnormalities that can be revealed by echocardiography, ventriculography, MRI scan and endomyocardial biopsy $[1,2]$. An RVOT is a tachycardia not combined with other cardiac diseases or obvious structural cardiac abnormalities [3]. The presence of epsilon wave (on 12lead ECG), spontaneous and induced episodes of VT, dilation of right ventricle (in echocardiography) and fibrosis with fatty tissue infiltration in MRI scan had an important role in the diagnosis of ARVD in our patient.

A first criterion for differentiation in the electrophysiological laboratory between two types of VT is the mode of induction: with $82 \%$ of the ARVD patients being inducible with ventricular extrastimuli in the study of O'Donnel et al. versus only 3\% in the RVOT group, indicating the reentrant mechanism of the VTs in the majority of patients with ARVD [4]. In our case the two VTs were induced by ventricular extrastimuli.

Secondly, O'Donnell et al. reported that electrophysiological study revealed more than one morphology of inducible VTs (a range of one to six VTs), with $71 \%$ of the patients having more than one morphology [4]. In contrast, the patients with RVOT all had only one morphology. The different morphologies in the ARVD group is best explained by the presence of an extensive arrhythmogenic substrate allowing the perpetuation of multiple circuits [5, 6]. We found three VT morphologies in the same patient: one spontaneous, and two induced during electrophysiological study originating from a right ventricle that showed extended damage at the MRI (apex, right ventricle free wall and right ventricular outflow tract).

The three VTs had different morphologies: two with LBBB pattern and one with RBBB pattern. As previously reported, RBBB can be observed during VT and does not exclude a right ventricular origin [6]. All RBBB morphology VTs in the paper of Miljoen et al. exhibited a peri-tricuspid circuit [6].

Several approaches have been proposed for treating VT in ARVD. Ablation rarely abolishes all arrhythmias in ARVD. The progressive nature of the disease and the acute success rate (reported to be lower than 40\%) makes ICD implantation a valuable alternative, shown to reduce mortality in ARVD [7]. Implantable cardioverter defibrillator therapy provides life-saving protection by effective termination of the tachycardia [8]. Our patient manifested one spontaneous VT that was well tolerated hemodynamically, and two induced in the electrophysiological lab: one pre-syncopal that was stopped by ventricular overdrive and one with hemodynamic intolerance that necessitated electrical shock for termination. The contribution of electrophysiological study in our patient allowed the decision to implant a cardioverter defibrillator for the prevention of sudden cardiac death.

\section{CONCLUSION}

According to this case and to other similar reports in medical literature, electrophysiological study in combination with other diagnostic tools such as magnetic resonance imaging, echocardiography and 12-lead electrocardiography becomes useful for the differential diagnosis between arrhythmogenic right ventricular dysplasia and right ventricular outflow tract tachycardia and contributes to decision of implantable cardioverter defibrillators implantation.

\section{$* * * * * * * * *$}

\section{Acknowledgements}

MD Gabriel Gusetu, MD Lucian Muresan, MD, PhD. Radu Rosu, MD. PuiuMihai, MD, PhD. Adina Malai, MD Mirela Cebanu, MD, PhD Chiroiu Bogdan, MD, Aurelia Gudal.

\section{Author Contributions}

Daniel Pop-Mandru - Substantial contributions to conception and design, Acquisition of data, Drafting the article, Revising it critically for important intellectual content, Final approval of the version to be published Gabriel Cismaru - Substantial contributions to conception and design, Acquisition of data, Drafting the article, Revising it critically for important intellectual content, Final approval of the version to be published Dana Pop - Substantial contributions to conception and design, Acquisition of data, Drafting the article, Revising it critically for important intellectual content, Final approval of the version to be published

Dumitru Zdrenghea - Substantial contributions to conception and design, Acquisition of data, Drafting the article, Revising it critically for important intellectual content, Final approval of the version to be published

\section{Guarantor}

The corresponding author is the guarantor of submission.

\section{Conflict of Interest}

Authors declare no conflict of interest.

\section{Copyright}

(C) Daniel Pop-Mandru et al. 2014; This article is distributed under the terms of Creative Commons attribution 3.0 License which permits unrestricted use, distribution and reproduction in any means provided the original authors and original publisher are properly credited. (Please see www.ijcasereportsandimages.com/ copyright-policy.php for more information.) 


\section{REFERENCES}

1. Corrado D, Basso C, Thiene G. Arrhythmogenic right ventricular cardiomyopathy: Diagnosis, prognosis, and treatment. Heart 2000;83(5):588-95.

2. McKenna WJ, Thiene G, Nava A, et al. Diagnosis of arrhythmogenic right ventricular dysplasia/ cardiomyopathy. Task Force of the Working Group on Myocardial and Pericardial Disease Of the European Society of Cardiology and the Scientific Council on Cardiomyopathies of the International Society and Federation of Cardiology. Br Heart J 1994;71(3):2158.

3. Klein LS, Shih HT, Hackett FK, Zipes DP, Miles WM. Radiofrequency catheter ablation of ventricular tachycardia in patients without structural heart disease. Circulation 1992;85(5):1666-74.

4. O’Donnell D, Cox D, Bourke J, Mitchell L, Furniss S. Clinical and electrophysiological differences between patients with arrhythmogenic right ventricular dysplasia and right ventricular outflow tract tachycardia. Eur Heart J 2003;24(9):801-10.
5. Katsouras GE, Margos PN, Livanis EG, Theodorakis GN, Kremastinos DT. Contribution of electroanatomical mapping to the diagnosis of arrhythmogenic right ventricular cardiomyopathy in a patient with sustained ventricular tachycardia. Hellenic J Cardiol 2006;47(3):184-9.

6. Hielko Miljoen, Simona State, Christian de Chillou, et al. Electroanatomic mapping characteristics of ventricular tachycardia in patients with arrhythmogenic right ventricular cardiomyopathy/ dysplasia. Europace 2005;7(6):516-24.

7. Fontaine G, Tonet J, Gallais Y, et al. Ventricular tachycardia catheter ablation in arrhythmogenic right ventricular dysplasia: A 16 year experience. Curr Cardiol Rep 2000;2(6):498-506.

8. Wichter T, Paul M, Wollmann C, et al. Implantable cardioverter/defibrillator therapy in arrhythmogenic right ventricular cardiomyopathy. Single center experience of long term follow-up and complications in 60 patients. Circulation 2004;109(12):1503-8.
Access full text article on other devices

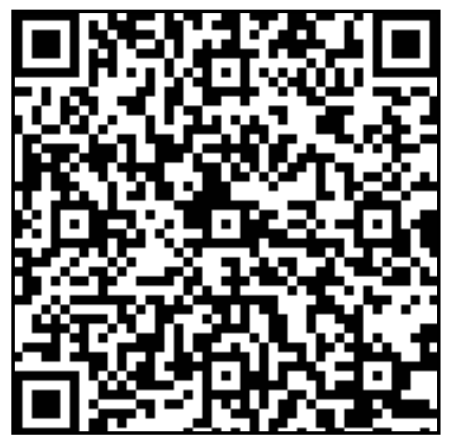

Access PDF of article on other devices

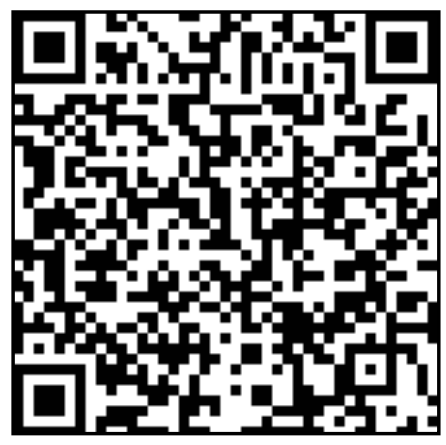

